

С. Ю. Шустиков, Критерий регулярности математической модели лазера, Матем. заметки, 2000, том 67, выпуск 5, 788-796

DOI: https://doi.org/10.4213/mzm896

Использование Общероссийского математического портала Math-Net.Ru подразумевает, что вы прочитали и согласны с пользовательским соглашением http://www . mathnet.ru/rus/agreement

Параметры загрузки:

IP : 54.166 .219 .16

26 апреля 2023 г., 14:54:52

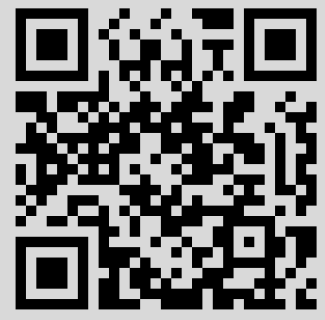




\title{
КРИТЕРИЙ РЕГУЛЯРНОСТИ МАТЕМАТИЧЕСКОЙ МОДЕЛИ ЛАЗЕРА
}

\author{
С. Ю. Шустиков
}

\begin{abstract}
Существует глубокая связь между условиями отсутствия взрыва для марковской эволюции в классической и квантовой теориях вероятностей. В обоих случаях эти условия эквивалентны сохранению единичного оператора (полной вероятности) минимальной марковской полугруппой. В настоящей работе изучается гайзенберговская эволюция модели, описывающей взаимодействие между цепочкой $N$ двухуровневых атомов и $n$-модовым внешним бозонным полем, рассмотренной недавно Дж. Алли и Дж. Сьюэллом. Неограниченный генератор марковской эволюции для наблюдаемых системы действует в алгебре фон Неймана. Доказано условие отсутствия взрыва для генератора марковской полугруппы, являющееся операторным аналогом подобного условия, предложенного Р.З. Хасьминским и, позже, Т. Танигучи для классических стохастических процессов. В случае операторных алгебр это условие влечет единственность и консервативность квантовой динамической полугруппы, описывающей марковскую эволюцию состояний системы. В регулярном случае условие отсутствия взрьва устанавливает однозначное соответствие между формальным генератором и инфинитезимальным оператором марковской полугруппы.
\end{abstract}

Библиографоия: 12 названий.

Предметом исследования данной работы является цепочка $N$ двухуровневых атомов, взаимодействуюших с $n$-модовым внешним полем [1]. Наблюдаемые системы представляют собой ограниченные операторы, действуюшие в гильбертовом пространстве $\mathscr{H}=\left(\bigotimes_{r=1}^{N} \mathbb{C}^{2}\right) \otimes \Gamma^{s}\left(\ell_{2}^{\otimes n}\right)$, где первый сомножитель в тензорном произведении отвечает пространству состояний "вещества" (т.е. цепочки атомов), а второй - пространству состояний “поля", а $\ell_{2}$ есть пространство квадратично-суммируемых последовательностей. Алгебра наблюдаемых, соответствующая веществу, есть $\bigotimes_{r=1}^{N} \mathbb{M}_{2}^{(r)}$, где через $\mathbb{M}_{2}^{(r)}$ обозначена алгебра комплексных $(2 \times 2)$-матриц, действующих на $r$-й сомножитель в тензорном произведении гильбертовых пространств. Элементами, порождающими алгебру наблюдаемых поля, являются бозонные операторы рождения-уничтожения, действующие в симметричном фоковском пространстве $\Gamma^{s}\left(\ell_{2}^{\otimes n}\right)$ с естественно определенным скалярньм произведением. Через $\mathscr{B}(\mathscr{H})$ обозначим алгебру фон Неймана всех ограниченных операторов.

Генератор эволюции в $\mathscr{B}(\mathscr{H})$ имеет стандартную линдбладовскую форму [2], [3]:

$$
\mathscr{L}(X)=\Phi(X)-G^{*} X-X G, \quad G=i H+\frac{1}{2} \Phi(\mathbb{I}),
$$

Работа выполнена при поддержке гранта INTAS 96-0698. 
где $X$ - некоторая наблюдаемая, а уравнение эволюции имеет вид

$$
\frac{d}{d t} P_{t}(X)=\mathscr{L}\left(P_{t}(X)\right),\left.\quad P_{t}(X)\right|_{t=0}=X, \quad X \in \mathscr{B}(\mathscr{H}),
$$

где $P_{t}(\cdot): \mathscr{B}(\mathscr{H}) \mapsto \mathscr{B}(\mathscr{H})$ называется квантовой динамической полугруппой [2], [3]. Пусть $\mathscr{T}(\mathscr{H})$ - алгебра всех операторов следового класса и $\mathscr{T}_{1}(\mathscr{H}) \subset \mathscr{T}(\mathscr{H})-$ конус всех положительных операторов с единичным следом. Для любого начального состояния $\rho \in \mathscr{T}_{1}(\mathscr{H})$ ожидание наблюдаемой $X$ в момент времени $t$ определяется операцией взятия следа

$$
\langle X\rangle_{t}=\operatorname{Tr} \rho P_{t}(X)
$$

В [4] доказано, что для широкого класса марковских процессов на $\mathbb{R}^{n}$ существуют естественные расширения их генераторов до генераторов квантовых динамических полугрупп $\mathscr{B}\left(\mathscr{L}_{2}\left(\mathbb{R}^{n}\right)\right)$ вида $(1)$, сохраняюших вышеуказанньй вид ожидания.

В соответствии с [1] вполне положительная компонента $\Phi(\cdot)$ генератора $\mathscr{L}(\cdot)$ (см. (1)) действует как $\Phi(A \otimes B)=\Phi_{\mathrm{f}}(A \otimes B)+\Phi_{\mathrm{a}}(A \otimes B)$, где "f" и “а" означают поле и атомы соответственно. Более подробно,

$$
\begin{aligned}
\Phi_{\mathrm{f}}(A \otimes B) & =\sum_{r=1}^{N} \sum_{l=0}^{n-1}\left(\varkappa_{l} A \otimes a_{l}^{\dagger} B a_{l}\right), \\
\Phi_{\mathrm{a}}(A \otimes B) & =\sum_{r=1}^{N} \sum_{l=0}^{n-1}\left(b_{z, r} \sigma_{z, r} A \sigma_{z, r}+b_{-, r} \sigma_{+, r} A \sigma_{-, r}+b_{+, r} \sigma_{-, r} A \sigma_{+, r}\right) \otimes B .
\end{aligned}
$$

Оператор $H=H_{\mathrm{f}}+H_{\mathrm{a}}+H_{\mathrm{int}}$, где "int" означает взаимодействие, является симметричным гамильтонианом с компонентами

$$
\begin{aligned}
H_{\mathrm{f}} & =\sum_{r=1}^{N} \sum_{l=0}^{n-1}\left(\omega_{l} \mathbb{I} \otimes a_{l}^{\dagger} a_{l}\right), \quad H_{\mathrm{a}}=\sum_{r=1}^{N} \sum_{l=0}^{n-1}\left(\frac{\epsilon}{2} \sigma_{z, r} \otimes \mathbb{I}\right), \\
H_{\mathrm{int}} & =\sum_{r=1}^{N} \sum_{l=0}^{n-1} \frac{i \lambda_{l}}{\sqrt{N}}\left(\sigma_{-, r} \otimes a_{l}^{\dagger} e^{-2 \pi i k_{l} r}-\sigma_{+, r} \otimes a_{l} e^{2 \pi i k_{l} r}\right) .
\end{aligned}
$$

В выражениях (3), (4) $A \in \bigotimes_{r=1}^{N} \mathbb{M}_{2}^{(r)}, B \in \mathscr{B}\left(\Gamma^{s}\left(\ell_{2}^{\otimes n}\right)\right), \sigma_{ \pm, r}=\sigma_{x, r} \pm i \sigma_{y, r}$, где $\sigma_{x, r}, \sigma_{y, r}, \sigma_{z, r}-$ стандартные матрицы Паули, действующие в пространстве состояний $r$-го атома, $b_{ \pm, r}>0, b_{z, r}>0, \lambda_{l}>0$, а операторы $a_{l}, a_{l}^{\dagger}$ удовлетворяют каноническим коммутационным соотношениям на $\ell_{2}^{\otimes n}:\left[a_{l}, a_{m}^{\dagger}\right]=\delta_{l m},\left[a_{l}, a_{m}\right]=0,\left[a_{l}^{\dagger}, a_{m}^{\dagger}\right]=0$.

Основные свойства генератора $\mathscr{L}(\cdot)$ и его компонент описьваются следующим предложением, где для упрощения записей положено $\mathbb{I} \in \mathscr{B}(\mathscr{H})$ вместо выражения $\mathbb{I} \otimes \mathbb{I}$, в котором первый и второй единичные операторы являются соответственно элементами алгебр наблюдаемых атомов и поля.

TEOPEMA 1. $\Pi p u \varkappa_{l}>0, \omega_{l}>0$

1) оператор $-G$ является генератором сильно непрерывной однопараметрической сжимающей голоморфной полугруппы на $\mathscr{H}$, а $\Phi(\mathbb{I})$ - самосопряжсенным оператором; 
2) $\mathscr{D} \stackrel{\text { def }}{=} \operatorname{dom} G^{m}$ является существенной областью определения оператора $\Phi(\mathbb{I}) n p u$ любом $m \geqslant 2$;

3) $\mathscr{D} \subseteq \operatorname{dom} \Phi(\mathbb{I}) \cap \operatorname{dom} H, H$ симметричен на $\mathscr{D}$, а единичный оператор действует в $\mathscr{H}$

4) отображсние $e^{-\varepsilon \Phi(\mathbb{I})} \Phi(\cdot) e^{-\varepsilon \Phi(\mathbb{I})}$ нормально и вполне полохсттельно при $\forall \varepsilon>0$

5) существует $c>0$ такое, что $\mathscr{L}(\Phi(\mathbb{I})) \leqslant c \Phi(\mathbb{I})$ в смысле квадратичных форм на $\mathscr{D}$.

Последнее условие является операторньм аналогом достаточных условий отсутствия взрыва, изученных Р.З. Хасьминским [5], Т. Танигучи [6], Д. У. Струком и С. Р. Вараданом [7].

ДоказАтЕльство. 1) Докажем следующее:

1. оператор $-G$ определен на плотном множестве и замкнут;

2. резольвентное множество $P(-G)$ содержит сектор $|\arg \lambda|\langle\pi / 2+\beta, \beta\rangle 0$;

3. для $\forall \varepsilon>0$ справедлива оценка

$$
\left\|(G+\lambda)^{-1}\right\| \leqslant \frac{M_{\varepsilon}}{|\lambda|}, \quad|\arg \lambda| \leqslant \frac{\pi}{2}+\beta-\varepsilon,
$$

где $M_{\varepsilon}$ не зависит от $\lambda$.

Если эти условия выполнены, оператор $-G$ является генератором полугрупшы $e^{-t G}$, которая голоморфна при $|\arg t|<\beta$, равномерно ограничена при $|\arg t| \leqslant \omega-\varepsilon$ и сильно непрерьвна в меньшем секторе при $t=0$ (см. [4]).

Представим оператор $G$ в виде разложения $G=G_{0}+G_{b}$ :

$$
\begin{aligned}
G_{0}= & \frac{1}{2} \Phi_{\mathrm{f}}(\mathbb{I})+H_{\mathrm{f}}=N \mathbb{I} \otimes \sum_{l=0}^{n-1}\left(\frac{1}{2} \varkappa_{l}+i \omega_{l}\right) a_{l}^{\dagger} a_{l}, \\
G_{b}= & \frac{1}{2} \Phi_{\mathrm{a}}(\mathbb{I})+i H_{\mathrm{a}}+i H_{\mathrm{int}} \\
= & \frac{n}{2} \sum_{r=1}^{N}\left(b_{-, r} \sigma_{+, r} \sigma_{-, r} \otimes \mathbb{I}+b_{+, r} \sigma_{-, r} \sigma_{+, r} \otimes \mathbb{I}+b_{z, r} \mathbb{I} \otimes \mathbb{I}\right) \\
& -\frac{1}{\sqrt{N}} \sum_{l=0}^{n-1} \sum_{r=1}^{N} \lambda_{l}\left(\sigma_{-, r} \otimes a_{l}^{\dagger} e^{-2 \pi i k_{l} r}-\sigma_{+, r} \otimes a_{l} e^{2 \pi i k_{l} r}\right)+i \frac{n \epsilon}{2} \sum_{r=1}^{N} \sigma_{z, r} \otimes \mathbb{I},
\end{aligned}
$$

где $\varkappa_{l}>0, \omega_{l}>0$, а оператор $G_{0}$ диагонален.

Для доказательства того, что $-G=-\left(G_{0}+G_{b}\right)$ является генератором, прежде всего докажем, что $-G_{0}$ есть генератор ограниченной голоморфной полугрупшы, а $G_{b}$ ограничен относительно $G_{0}$ с нулевой относительной верхней гранью. Согласно [4] этого достаточно, чтобы $-G$ порождал голоморфную полугруппу.

Прежде всего установим, что $G_{0}$ замкнут на области

$$
\operatorname{dom} G_{0}=\left\{\Psi:\|\Psi\|_{\mathscr{H}}^{2}+\left\|G_{0} \Psi\right\|_{\mathscr{H}}^{2}<\infty\right\}
$$


С этой целью проверим эквивалентное утверждение о том, что для любого $\lambda>0$ оператор $G_{0}+\lambda \mathbb{I}$ замкнут. Применим критерий из [4] для оператора $G_{0}$, определенного на линейной оболочке экспоненциальных векторов. Согласно этому критерию оператор $G_{0}$ имеет замькание тогда и только тогда, когда $\lim _{N \rightarrow \infty} \Psi_{N}=0, \Psi_{N} \in \operatorname{dom} G_{0}$ и $\lim _{N \rightarrow \infty} G_{0} \Psi_{N}=\widetilde{\Psi}$ влечет $\widetilde{\Psi}=0$.

Рассмотрим вектор $\varphi_{N}=\left(G_{0}+\lambda \mathbb{I}\right) \Psi_{N}$, где последовательность $\left\{\Psi_{N}\right\}$ такова, что

$$
\lim _{N \rightarrow \infty}\left\|\Psi_{N}\right\|=0, \quad \Psi_{N} \in \operatorname{dom} G_{0}, \quad G_{0} \Psi_{N} \rightarrow \widetilde{\Psi}
$$

Таким образом, имеем $\lim _{N \rightarrow \infty} \varphi_{N}=\widetilde{\Psi}, \Psi_{N}=\left(G_{0}+\lambda \mathbb{I}\right)^{-1} \varphi_{N}$. Справедлива следующая оценка для нормы:

$$
\left\|\left(G_{0}+\lambda \mathbb{I}\right)^{-1} \varphi_{N}\right\| \leqslant\left\|\left(G_{0}+\lambda \mathbb{I}\right)^{-1}\left(\widetilde{\Psi}-\varphi_{N}\right)\right\|+\left\|\left(G_{0}+\lambda \mathbb{I}\right)^{-1} \varphi_{N}\right\| .
$$

Определение сходимости дает

$$
\begin{aligned}
& \left\{\lim _{N \rightarrow \infty} \varphi_{N}=\widetilde{\Psi}\right\} \Longleftrightarrow\left\{\forall \varepsilon_{1}>0 \quad \exists N_{1}\left(\varepsilon_{1}\right): \forall N>N_{1}\left(\varepsilon_{1}\right)\left\|\widetilde{\Psi}-\varphi_{N}\right\|<\varepsilon_{1}\right\}, \\
& \left\{\lim _{N \rightarrow \infty}\left(G_{0}+\lambda \mathbb{I}\right)^{-1} \varphi_{N}=0\right\} \\
& \Longleftrightarrow\left\{\forall \varepsilon_{2}>0 \quad \exists N_{2}\left(\varepsilon_{2}\right): \quad \forall N>N_{2}\left(\varepsilon_{2}\right)\left\|\left(G_{0}+\lambda \mathbb{I}\right)^{-1} \varphi_{N}\right\|<\varepsilon_{2}\right\} .
\end{aligned}
$$

Положим $\widetilde{N}=\max \left\{N_{1}, N_{2}\right\}$. Для $\forall N>\widetilde{N}$ справедлива оценка

$$
\begin{aligned}
\left\|\left(G_{0}+\lambda \mathbb{I}\right)^{-1}\left(\widetilde{\Psi}-\varphi_{N}\right)\right\| & \leqslant \sqrt{\sum_{k=0}^{\infty}\left|\frac{1}{\sum_{l=0}^{n-1}\left(\frac{1}{2} \varkappa_{l}+i \omega_{l}\right) k+\lambda}\right| \widetilde{\Psi}_{k}-\left.\left.\varphi_{N, k}\right|^{2}\right|^{2}} \\
& \leqslant \frac{1}{\lambda} \sqrt{\sum_{k=0}^{\infty}\left|\widetilde{\Psi}_{k}-\varphi_{N, k}\right|^{2}}=\frac{1}{\lambda}\left\|\widetilde{\Psi}-\varphi_{N}\right\| .
\end{aligned}
$$

Как прямое следствие, получаем

$$
\left\|\left(G_{0}+\lambda \mathbb{I}\right)^{-1} \varphi_{N}\right\|<\frac{1}{\lambda} \varepsilon_{1}+\varepsilon_{2}
$$

Так как $\widetilde{\Psi}$ не зависит от $\widetilde{N},\left(G_{0}+\lambda \mathbb{I}\right)^{-1} \varphi_{N}=0$. Так как ядро оператора $\left(G_{0}+\lambda \mathbb{I}\right)^{-1}$ тривиально, имеем $\widetilde{\Psi}=0$ и $\left(G_{0}+\lambda \mathbb{I}\right)$ замкнут.

Множество $\operatorname{dom} G_{0}$ очевидным образом замкнуто в $\mathscr{H}$, так как $G_{0}$ определен на тотальном множестве экспоненциальньх векторов, линейная оболочка которых замыкаема в норме графика оператора $G_{0}$.

Рассмотрим свойства резольвентного оператора $\mathfrak{R}\left(-G_{0}, \lambda\right)=-\left(G_{0}+\lambda\right)^{-1}$. Отрицательная вещественная полуось принадлежит резольвентному множеству оператора $\varkappa_{l} a_{l}^{\dagger} a_{l}$, так как все коэффициенты $\varkappa_{l}$ положительны (это энергии квантов поля), а операторы числа частиц $a_{l}^{\dagger} a_{l}$ имеют неотрицательные собственные значения. 
Для доказательства неравенства (5) перепишем резольвенту диагонального оператора $-G_{0}$ в интегральной форме

$$
\mathfrak{R}\left(-G_{0}, \lambda\right)=\int_{0}^{\infty} e^{-\lambda t} e^{-G_{0} t} d t
$$

и оценим ее норму.

Введем обозначения

$$
\varkappa=\sum_{l=0}^{n-1} \varkappa_{l}, \quad \omega=\sum_{l=0}^{n-1} \omega_{l}, \quad \lambda=|\lambda| e^{i \varphi}, \quad \varphi \in\left[-\frac{\pi}{2}-\beta+\varepsilon, \frac{\pi}{2}+\beta-\varepsilon\right] .
$$

Имеем

$$
\left\|\Re\left(-G_{0}, \lambda\right) \Psi\right\|^{2}=\sum_{k=0}^{\infty} \frac{\left\|\Psi_{k}\right\|^{2}}{\left(|\lambda| \cos \varphi+\frac{1}{2} \varkappa k\right)^{2}+(|\lambda| \sin \varphi+\omega k)^{2}}
$$

где $\Psi=\Psi_{0} \oplus \Psi_{1} \oplus \cdots \oplus \Psi_{k} \oplus \cdots \in \Gamma^{s}\left(\ell_{2}^{\otimes n}\right)$. Положим

$$
\begin{aligned}
\Lambda & \stackrel{\text { def }}{=}\left(|\lambda| \cos \varphi+\frac{1}{2} \varkappa k\right)^{2}+(|\lambda| \sin \varphi+\omega k)^{2} \\
& =|\lambda|^{2}+\frac{1}{4} k^{2}\left(\varkappa^{2}+4 \omega^{2}\right)+|\lambda| k(\varkappa \cos \varphi+2 \omega \sin \varphi), \\
f(\varphi) & \stackrel{\text { def }}{=} \varkappa \cos \varphi+2 \omega \sin \varphi .
\end{aligned}
$$

Наша цель - отыскать такое $\alpha>0$, что $\Lambda \geqslant \alpha|\lambda|^{2}$. Вычислим экстремумы функции $f(\varphi)$. Условие $f^{\prime}(\varphi)=0$ дает

$$
\varphi_{\max }=\operatorname{arctg} \frac{2 \omega}{\varkappa}, \quad \varphi_{\min }=\operatorname{arctg} \frac{2 \omega}{\varkappa}+\pi
$$

Абсолютньй минимум $\varphi_{\min }$ может принадлежать сектору, в котором рассматривается резольвента. В этом случае

$$
\begin{gathered}
\Lambda=|\lambda|^{2}+\frac{1}{4} k^{2}\left(\varkappa^{2}+4 \omega^{2}\right)+|\lambda| k\left(\varkappa \cos \varphi_{\min }+2 \omega \sin \varphi_{\min }\right) \geqslant \alpha|\lambda|^{2} \\
(1-\alpha) \frac{|\lambda|^{2}}{k^{2}}-\frac{|\lambda|}{k} \sqrt{\varkappa^{2}+4 \omega^{2}}+\frac{1}{4}\left(\varkappa^{2}+4 \omega^{2}\right) \geqslant 0 .
\end{gathered}
$$

Это неравенство справедливо для всех $|\lambda|$ и $k$, если дискриминант квадратного трехчлена неположителен: $D=\varkappa^{2}+4 \omega^{2}-(1-\alpha)\left(\varkappa^{2}+4 \omega^{2}\right)=\alpha\left(\varkappa^{2}+4 \omega^{2}\right) \leqslant 0$. Но $\alpha>0$, поэтому абсолютньй минимум функции $f(\varphi)$ должен лежать вне сектора (иначе невозможно написать требуемую оценку для резольвенты), т.е.

$$
\operatorname{tg}(\beta-\varepsilon)<\frac{\varkappa}{2 \omega}
$$


Значения $f(\varphi)$ на границах сектора равны

$$
\begin{aligned}
f\left(\frac{\pi}{2}+\beta-\varepsilon\right) & =\varkappa \cos \left(\frac{\pi}{2}+\beta-\varepsilon\right)+2 \omega \sin \left(\frac{\pi}{2}+\beta-\varepsilon\right) \\
& =-\varkappa \sin (\beta-\varepsilon)+2 \omega \cos (\beta-\varepsilon), \\
f\left(-\left(\frac{\pi}{2}+\beta-\varepsilon\right)\right) & =\varkappa \cos \left(-\left(\frac{\pi}{2}+\beta-\varepsilon\right)\right)+2 \omega \sin \left(-\left(\frac{\pi}{2}+\beta-\varepsilon\right)\right) \\
& =-\varkappa \sin (\beta-\varepsilon)-2 \omega \cos (\beta-\varepsilon),
\end{aligned}
$$

причем второе является абсолютньм минимумом в секторе. После подстановки его в выражение для $\Lambda$ получаем следующую оценку для $\alpha$ :

$$
\alpha \leqslant \frac{(\varkappa \cos (\beta-\varepsilon)-2 \omega \sin (\beta-\varepsilon))^{2}}{\varkappa^{2}+4 \omega^{2}}
$$

справедливую, если условие (7) вьполнено. Так как $\varepsilon>0$ произвольно мало, имеем $\operatorname{tg}(\beta)<\varkappa /(2 \omega)$, где $\beta>0$ определяет сектор $(-\pi / 2-\beta, \pi / 2+\beta)$, в котором справедливы оценки $\Lambda \geqslant \alpha|\lambda|^{2}$ и

$$
\left\|\mathfrak{R}\left(-G_{0}, \lambda\right) \Psi\right\|^{2} \leqslant \frac{1}{\alpha|\lambda|^{2}} \sum_{k=0}^{\infty}\left\|\Psi_{k}\right\|^{2}=\frac{1}{\alpha|\lambda|^{2}}\|\Psi\|^{2}
$$

Требование (7) не существенно, так как для любых ограниченных $\varkappa$ и $\omega$ можно выбрать $\beta$ достаточно малым и построить полугруппу при помощи интеграла Данфорда-Тейлора

$$
e^{-t G_{0}}=\frac{1}{2 \pi i} \int_{\Gamma} e^{\lambda t}\left(G_{0}+\lambda\right)^{-1} d \lambda
$$

где $\Gamma$ - кривая, лежашая целиком в секторе $(-\pi / 2-\beta, \pi / 2+\beta)$, идущая из бесконечности с $\arg \lambda=-(\pi / 2+\beta-\varepsilon)$ и уходящая на бесконечность $\mathrm{c} \arg \lambda=\pi / 2+\beta-\varepsilon$. Следовательно, $-G_{0}$ является генератором голоморфной полугрупшы.

Докажем, что $G_{b}$ ограничен относительно $G_{0}$ с произвольно малой относительной верхней границей. Для этого применим известное операторное неравенство

$$
A^{*} B+B^{*} A \leqslant \varepsilon A^{*} A+\varepsilon^{-1} B^{*} B \quad \forall \varepsilon>0
$$

в форме

$$
\sum_{l=0}^{n-1}\left(A_{l}^{*} \otimes B_{l}+B_{l}^{*} \otimes A_{l}\right) \leqslant \varepsilon \sum_{l=0}^{n-1} A_{l}^{*} A_{l} \otimes \mathbb{I}+\varepsilon^{-1} \sum_{l=0}^{n-1} \mathbb{I} \otimes B_{l}^{*} B_{l} \quad \forall \varepsilon>0
$$

где положим $A_{l}=a_{l}$,

$$
B_{l}=\frac{\lambda_{l}}{\sqrt{N}} \sum_{r=1}^{N} \sigma_{-, r} e^{-2 \pi i k_{l} r}
$$


В выражении для $G_{b}$ (см. (6)) неограниченньми являются только слагаемые, содержашие $a_{l}$ и $a_{l}^{\dagger}$. Найдем их верхнюю гранищу относительно оператора $G_{0}$. Разложим $G_{b}$ на сумму $G_{b}=\widetilde{G}_{b}+g_{b}$, где

$$
\begin{aligned}
\widetilde{G}_{b} & =-\frac{1}{\sqrt{N}} \sum_{l=0}^{n-1} \sum_{r=1}^{N} \lambda_{l}\left(\sigma_{-, r} \otimes a_{l}^{\dagger} e^{-2 \pi i k_{l} r}-\sigma_{+, r} \otimes a_{l} e^{2 \pi i k_{l} r}\right), \\
g_{b} & =\frac{n}{2} \sum_{r=1}^{N}\left(b_{-, r} \sigma_{+, r} \sigma_{-, r} \otimes \mathbb{I}+b_{+, r} \sigma_{-, r} \sigma_{+, r} \otimes \mathbb{I}+b_{z, r} \mathbb{I} \otimes \mathbb{I}+i \frac{\epsilon}{2} \sigma_{z, r} \otimes \mathbb{I}\right) .
\end{aligned}
$$

Заметим, что $\left\|g_{b}\right\|<\infty$. Из неравенства треугольника и из (6) имеем

$$
\|a+b\|^{2} \leqslant 2\|a\|^{2}+2\|b\|^{2}, \quad\left\|G_{b} \Psi\right\|^{2} \leqslant 2\left\|\widetilde{G}_{b} \Psi\right\|^{2}+2\left\|g_{b} \Psi\right\|^{2} .
$$

Применяя канонические коммутационные соотношения для $a_{l}, a_{l}^{\dagger}$, получим

$$
\left\|\widetilde{G}_{b} \Psi\right\|^{2}=\frac{1}{N} \sum_{l=0}^{n-1} \sum_{r=1}^{N} \lambda_{l}^{2}\left(\left\|a_{l}^{\dagger} \Psi\right\|^{2}+\left\|a_{l}^{\dagger} \Psi\right\|^{2}\right)=\frac{1}{N} \sum_{l=0}^{n-1} \sum_{r=1}^{N} \lambda_{l}^{2}\left(2\left\|a_{l} \Psi\right\|^{2}+\|\Psi\|^{2}\right),
$$

где $\left\|a_{l} \Psi\right\|^{2}=\left\langle\Psi, a_{l}^{\dagger} a_{l} \Psi\right\rangle$.

Так как $\mathbb{I} \cdot a_{l}^{\dagger} a_{l}+a_{l}^{\dagger} a_{l} \cdot \mathbb{I} \leqslant \varepsilon^{-1} \mathbb{I}+\varepsilon\left(a_{l}^{\dagger} a_{l}\right)^{2}$, имеем $\left\|a_{l} \Psi\right\|^{2} \leqslant \varepsilon^{-1}\|\Psi\|^{2}+\varepsilon\left\|a_{l}^{\dagger} a_{l} \Psi\right\|^{2}$ на $\operatorname{dom} G_{0}$. Следовательно, оператор $\widetilde{G}_{b}$ ограничен относительно $G_{0}$ :

$$
\begin{aligned}
\left\|\widetilde{G}_{b} \Psi\right\|^{2} & \leqslant \frac{1}{N} \sum_{l=0}^{n-1} \sum_{r=1}^{N} \lambda_{l}^{2}\left(2\left\|a_{l} \Psi\right\|^{2}+\|\Psi\|^{2}\right) \\
& \leqslant \frac{1}{N} \sum_{l=0}^{n-1} \sum_{r=1}^{N} \lambda_{l}^{2}\left(2 \varepsilon^{-1}\|\Psi\|^{2}+2 \varepsilon\left\|a_{l}^{\dagger} a_{l} \Psi\right\|^{2}+\|\Psi\|^{2}\right) \\
& \leqslant \frac{1}{N} \sum_{l=0}^{n-1} \sum_{r=1}^{N} \lambda_{l}^{2}\left(\left(2 \varepsilon^{-1}+1\right)\|\Psi\|^{2}+2 \varepsilon \frac{1}{N \min _{l}\left|\frac{1}{2} \varkappa_{l}+i \omega_{l}\right|}\left\|G_{0} \Psi\right\|^{2}\right) .
\end{aligned}
$$

Искомая относительная верхняя грань равна нулю, так как

$$
\left\|G_{b} \Psi\right\|^{2} \leqslant \delta\left\|G_{0} \Psi\right\|^{2}+\delta^{-1}\|\Psi\|^{2}
$$

при произвольно малом $\delta$.

Следующий шаг состоит в применении теоремы (см. [4]), утверждающей, что операторы $G_{0}$ и $G$ замыкаемы или незамыкаемы одновременно и что их области определения совпадают.

Докажем самосопряженность оператора $\Phi(\mathbb{I})$. Слагаемые с $\sigma$-матрищами являются самосопряженными, так как они действуют в конечномерном пространстве $\bigoplus_{r=1}^{N} \mathbb{C}^{2}$. Остается доказать самосопряженность $T=\sum_{l} \varkappa_{l} a_{l}^{\dagger} a_{l}$. Он симметричен ввиду своей структуры, т.е. $T^{*} \supset T$, и имеет плотную область определения (линейную оболочку экспоненциальньх векторов), с которой может быть продолжен до своего замькания. Полученное расширение будет самосопряженным $\left(T^{*}=T\right)$, так как уравнение $T^{*} \Psi= \pm i \Psi$ не имеет ограниченных решений (см. [8], [9]). 
2) Доказательство того, что $\operatorname{dom} G_{0}^{m}=\operatorname{dom} G^{m}$, аналогично предыдущему пункту, так как $G_{0}^{m}$ является доминирующей частю оператора $G^{m}$. Область определения $G_{0}^{m}$ можно описать как

$$
\operatorname{dom} G_{0}^{m}=\mathbb{C}^{2} \otimes\left(\mathbb{I}+\sum_{l=0}^{n-1}\left|\frac{1}{2} \varkappa_{l}+i \omega_{l}\right| a_{l}^{\dagger} a_{l}\right)^{-m} \Gamma^{s}\left(\ell_{2}^{\otimes n}\right) .
$$

Векторы из $\operatorname{dom} G_{0}^{m}$ принадлежат областям определения $\Phi(\mathbb{I})$ и $H$, так как могут быть представлены в виде линейных комбинаций экспоненциальных векторов. Оператор $\Phi(\mathbb{I})$ обладает единственным самосопряженным расширением с произвольного плотного множества собственной области определения, которое обозначим также через $\Phi(\mathbb{I})$. Следовательно, $\operatorname{dom} G^{m}=\operatorname{dom} G_{0}^{m}=\operatorname{core} \Phi(\mathbb{I}) \in \operatorname{core} \Phi(\mathbb{I})^{1 / 2}$ при любом $m \geqslant 1$.

3) Условие $\mathscr{D} \subseteq \operatorname{dom} \Phi(\mathbb{I}) \cap \operatorname{dom} H$ доказано в предыдущем пункте, а оператор $H$ симметричен в $\mathscr{D}$ благодаря своей эрмитовой структуре.

4) Данное условие справедливо для класса нормальных вполне положительных отображений, имеющих краусову структуру (см.[10]), если $\Phi(\mathbb{I})$ корректно определен.

5) Для доказательства последнего условия необходимо оценить $\mathscr{L}(\Phi(\mathbb{I}))$. Используя канонические коммутационные соотношения, получим

$$
\begin{aligned}
& \mathscr{L}(\Phi(\mathbb{I}))=\Phi(\Phi(\mathbb{I}))-\Phi(\mathbb{I})^{2}+i[H, \Phi(\mathbb{I})] \\
& \quad=-N^{2} \mathbb{I} \otimes \sum_{l=0}^{n-1} \varkappa_{l}^{2} a_{l}^{\dagger} a_{l}+n^{2} \sum_{r=1}^{N} b_{+, r}\left(b_{-, r}-b_{+, r}\right) i \sigma_{y r} \otimes \mathbb{I} \\
& \quad+\frac{1}{\sqrt{N}} \sum_{r=1}^{N} \sum_{l=0}^{n-1} \lambda_{l}\left(N \varkappa_{l}-n\left(b_{-, r}-b_{+, r}\right)\right)\left(\sigma_{-, r} \otimes a_{l}^{\dagger} e^{-2 \pi i k_{l} r}+\sigma_{+, r} \otimes a_{l} e^{2 \pi i k_{l} r}\right) .
\end{aligned}
$$

Оценим двойную сумму, используя неравенство (8):

$$
\begin{aligned}
\sigma_{-, r} \otimes a_{l}^{\dagger} e^{-2 \pi i k_{l} r}+\sigma_{+, r} \otimes a_{l} e^{2 \pi i k_{l} r} & \leqslant \varepsilon \mathbb{I} \otimes a_{l}^{\dagger} a_{l}+\varepsilon^{-1} \sigma_{+, r} \sigma_{-, r} \otimes \mathbb{I} \\
& =\varepsilon \mathbb{I} \otimes a_{l}^{\dagger} a_{l}+\varepsilon^{-1} \frac{1}{2}\left(\mathbb{I}+\sigma_{z, r}\right) \otimes \mathbb{I} .
\end{aligned}
$$

Положим $b_{\max }=\max _{r}\left\{b_{+, r}, b_{-, r}, b_{z, r}\right\}, b_{\min }=\min _{r}\left\{b_{+, r}, b_{-, r}, b_{z, r}\right\}, \varkappa_{\max }=\max _{l} \varkappa_{l}$, $\varkappa_{\min }=\min _{l} \varkappa_{l}, \lambda_{\max }=\max _{l}\left|\lambda_{l}\right|$. Все эти величины конечны и положительны, так как имеют очевидный физический смысл интенсивностей переходов, энергий квантов и констант связи. Имеем $\left|b_{+, r}\left(b_{-, r}-b_{+, r}\right)\right| \leqslant 2 b_{\max }^{2},\left|b_{-, r}-b_{+, r}\right| \leqslant 2 b_{\max }$. Используя $\left\|i \sigma_{y}\right\|=1,\left\|\mathbb{I}+\sigma_{z, r}\right\|=2$, получим

$$
\begin{aligned}
\mathscr{L}(\Phi(\mathbb{I})) \leqslant & -N^{2} \varkappa_{\min }^{2} \mathbb{I} \otimes \sum_{l=0}^{n-1} a_{l}^{\dagger} a_{l}+\sqrt{N} \varepsilon \lambda_{\max }\left(N \varkappa_{\max }+2 n b_{\max }\right) \sum_{l=0}^{n-1} a_{l}^{\dagger} a_{l} \\
& +2 N n^{2} b_{\max }^{2} \mathbb{I}+\sqrt{N} 2 \varepsilon n \lambda_{\max }\left(N \varkappa_{\max }+2 n b_{\max }\right) \mathbb{I} .
\end{aligned}
$$

Первая и вторая суммы вместе могут быть ограничены сверху нулем, если выбрать $\varepsilon \leqslant\left(N^{3 / 2} \varkappa_{\min }^{2}\right) /\left(\lambda_{\max }\left(N \varkappa_{\max }+2 n b_{\max }\right)\right)$. Следовательно, для третьего и четвертого членов получаем неравенство

$$
2 N n^{2} b_{\max }^{2}+\sqrt{N} 2 \varepsilon n \lambda_{\max }\left(N \varkappa_{\max }+2 n b_{\max }\right) \leqslant 2 N n\left(n b_{\max }^{2}+N \varkappa_{\min }^{2}\right) .
$$


Используя выражения $\left\|\Phi_{\mathrm{a}}(\mathbb{I})\right\| \geqslant 3 N n b_{\min }$ и $\Phi_{\mathrm{a}}(\mathbb{I}) \leqslant \Phi(\mathbb{I})($ см. $(3))$, получим оценку

$$
\mathscr{L}(\Phi(\mathbb{I})) \leqslant c \Phi(\mathbb{I}) \quad \text { при } c \geqslant \frac{2}{3 b_{\min }}\left(n b_{\max }^{2}+N \varkappa_{\min }^{2}\right) \text {. }
$$

Это завершает доказательство того, что класс генераторов $(1),(3),(4)$, описывающих взаимодействие между веществом и полем, удовлетворяет критерию регулярности.

С математической точки зрения свойство регулярности генератора $\mathscr{L}(\cdot)$ важно потому, что в регулярном случае он определяет единственную консервативную квантовую динамическую полугруппу, являющуюся минимальным решением эволюционного уравнения (2). Это свойство представляет собой операторньй аналог свойства регулярности генераторов невзрьвных стохастических процессов. Используя достаточный критерий регулярности (см. [11], [12]) и теорему 1, получаем следующее утверждение.

ТЕОРема 2. При любом выборе ограниченных коэффичиентов $\varkappa_{l}, \omega_{l}, \lambda_{l}(l=0, \ldots$, $n-1), b_{+, r}, b_{-, r}, b_{z, r}(r=0, \ldots, N)$ u $\epsilon$ в формулах (3), (4) минимальная динамическая полугруппа $P_{t}(\cdot)$ с формальным генератором (1) консервативна. Она является единственным решением уравнения (2) в классе квантовых динамических nолугрупn.

Автор выражает благодарность профессору А. М. Чеботареву за помощь, оказанную при подготовке настоящей работы.

\section{СПИСОК ЦИТИРОВАННОЙ ЛИТЕРАТУРЫ}

[1] Alli G., Sewell G. New methods and structures in the theory of the multi-mode Dicke laser model // J. Math. Phys. 1995. V. 36. № 10. P. 5598-5626.

[2] Lindblad G. On generators of quantum dynamical semigroups // Comm. Math. Phys. 1976. V. 48. P. 119-130.

[3] Goriny V., Kossakowski A., Sudarshan E. C. G. Completely positive dynamical semigroups of $N$-level systems // J. Math. Phys. 1976. V. 17. P. 821-825.

[4] Kato T. Perturbation Theory of Linear Operators. New York: Springer Verlag, 1966.

[5] Khas'minskii R. Z. Ergodic problems of recurrent diffusion processes // Prob. Theory Appl. 1960. V. 5. №1. P. 196-214.

[6] Taniguchi T. On sufficient conditions for nonexplosion of solutions to stochastic differential equations // J. Math. Anal. Appl. 1990. V. 153. № 2. P. 549-561.

[7] Strook D. W., Varadhan S. R. Multidimensional Diffusion Processes. New York: Springer Verlag, 1979.

[8] Рид М., Саймон Б. Методы современной математической физики. Т. 1. М.: Мир, 1977.

[9] Березин Ф. А. Метод вторичного квантования. М.: Наука, 1986.

[10] Kraus K. General state changes in quantum theory // Ann. Phys. 1971. V. 64. P. 311-335.

[11] Chebotarev A. M. Necessary and sufficient conditions for conservativity of a dynamical semigroup // J. Soviet Math. 1991. V. 56. № 5. P. 2697-2719.

[12] Chebotarev A. M., Fagnola F. Sufficient conditions for conservativity of quantum dynamical semigroups // J. Funct. Anal. 1998. V. 153. № 2. P. 382-404.

Московский государственный университет им. М.В. Ломоносова

E-mail: shust@qs.phys.msu.su 